\title{
Manoeuvring Trials with the MUN Explorer AUV: Data Analysis and Observations
}

\author{
Manoj T. Issac ${ }^{1}$, Sara Adams², Moqin $\mathrm{He}^{2}$, Neil Bose ${ }^{3}$, \\ Christopher D. Williams ${ }^{4}$, Ralf Bachmayer ${ }^{4}$ \\ Tristan Crees ${ }^{5}$ \\ ${ }^{1}$ Ocean Engineering Research Centre (OERC), Memorial University of Newfoundland (MUN) \\ St. John's, NL, A1B 3X5, Canada \\ ${ }^{2}$ Research Lab Coordinators, MERLIN, Memorial University of Newfoundland (MUN) \\ St. John's, NL, A1B 3X5, Canada \\ ${ }^{3}$ Professor of Maritime Hydrodynamics and Manager, Australian Maritime Hydrodynamics Research Centre, \\ Australian Maritime College, Launceston, Tasmania 7250, Australia \\ (Formerly Professor, Canada Research Chair in Offshore and Underwater Vehicles \\ Memorial University of Newfoundland) \\ ${ }^{4}$ Research Engineers, National Research Council of Canada, Institute for Ocean Technology \\ St. John's, NL, A1B 3T5, Canada \\ ${ }^{5}$ Robotics Engineer, International Submarine Engineering Ltd, 1734 Broadway Street \\ Port Coquitlam, BC, V3C 2M8, Canada
}

\begin{abstract}
Manoeuvring trials are usually performed to determine the manoeuvring characteristics of a marine vehicle. It is through certain standard manoeuvres we evaluate the robustness, performance and limitations of the vehicle control system. A series of open-water manoeuvring trials were performed using the MUN Explorer AUV in the summer of 2006. The actual purpose of these experiments was to collect a set of experimental data in order to validate a hydrodynamic model of the dynamic performance of the vehicle. This paper presents the results and observations from the analysis of a set of manoeuvring trials data: in particular the results from straightline (acceleration - deceleration) tests and turning circles. It outlines briefly the method by which these tests were conducted and discusses the results and observations made. Apart from providing a data set for validation purposes, the results also indicate the ability of the vehicle to follow a pre-planned mission with precision.
\end{abstract}

\section{INTRODUCTION}

Autonomous Underwater Vehicles (AUVs) are selfpropelled robotic platforms that can perform a predetermined mission completely unmanned. The scientific demand for high-resolution spatial and temporal ocean observation data is increasing. The potential of AUV technology in undersea surveys and the significant improvement in the quality of data acquired using these platforms, compared to traditional ocean observation platforms like ships and buoys, is leading to increased use of AUVs. Also, these small, intelligent platforms potentially allow continuous surveying of ocean floor at lower cost, compared to a ship.

During a mission, an AUV may undergo different manoeuvring scenarios such as a complete turn at the end of a survey line, a severe turn during obstacle avoidance or frequent depth changes while following a rugged seabed terrain. This demands a high degree of manoeuvrability in order to achieve good position and attitude control. The designers of AUVs increasingly rely on computer models (hydrodynamic models) to study the motion behaviours and in turn to develop vehicle controllers.

Hydrodynamic models are often developed to predict the performance of these vehicles, especially their interaction with the fluid. A reliable model is one that can predict the actual vehicle response. A dynamics model of an axisymmetric streamlined AUV based on the "body-build-up" method of Nahon [1] was developed by Perrault [2] and Evans [3] using MATLAB ${ }^{\mathrm{TM}}$ and SIMULINK ${ }^{\mathrm{TM}}$ and is available for use at Memorial University of Newfoundland (MUN). In order to validate this model, it was necessary to have experimental data from real vehicles. The availability of the MUN Explorer AUV facilitated the experimental data collection. As a result, a series of manoeuvring trials was performed in 2006 at Holyrood, Newfoundland.

MUN acquired the survey-class AUV - the MUN Explorer, in June 2006. It was built by International Submarine Engineering (ISE) in Port Coquitlam, British Columbia. The primary areas of research for which the vehicle is intended to be utilized are environmental monitoring, seabed surveys etc.

The MUN Explorer AUV is a survey-class AUV that functions mainly as a sensor platform for undersea data collection purposes. The AUV is $4.5 \mathrm{~m}$ in length with a maximum mid-body diameter of $0.69 \mathrm{~m}$. The parallel mid-body section has a semi-ellipsoidal nose section at its front end and a faired tail section at its aft end that gives the vehicle a hydrodynamically efficient streamlined shape. The vehicle weighs $630 \mathrm{~kg}$ in air and is rated for $3000 \mathrm{~m}$ depth. The 
pressure hull, a ring stiffened cylindrical module cast from 7075 aluminium alloy, provides the majority of the buoyancy for the vehicle and space for dry components such as batteries and control electronics; the remainder of the hull is free-flooding. It can achieve a maximum operating speed of $2.5 \mathrm{~m} / \mathrm{s}$ using a twin-bladed propeller. Manoeuvring in 3-D space is facilitated by six control planes - two dive planes for precise depth control and four tail planes to control the roll, pitch and yaw motions of the vehicle. The vehicle is equipped with a retractable communications mast at the aft end, which can extend up to one metre above the body in order to reach out of the water to take position fixes when the vehicle climbs near the ocean surface; the antenna retracts back into the body automatically when the vehicle submerges. A detailed description of the various features of the vehicle, the equipments, sensors and other instruments it uses can be found in [4]. Figure 1 below shows the MUN Explorer AUV on the wharf ready to be launched.

A network of dock-side computers provide mission planning, monitoring and access to certain positioning sensors. They also send pilot commands to the vehicle and generate graphics, texts and diagnostics displays to provide information to the operator through a custom designed graphical user interface. All data collected during the tests from all the on-board sensors are acquired by the Vehicle Control Computer (VCC). The data collected on the VCC can later be retrieved by the dock-side console.

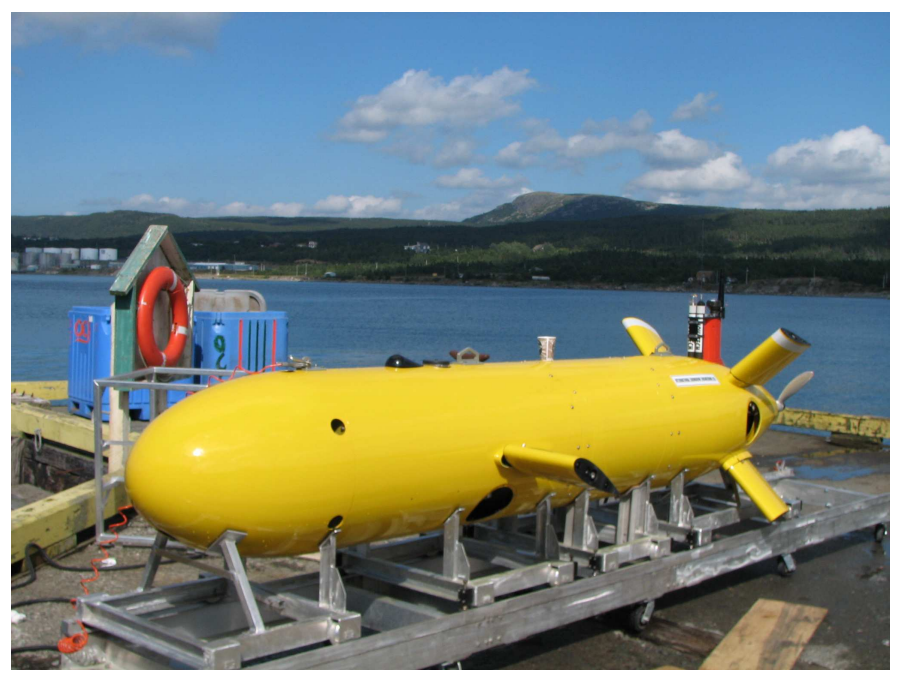

Fig. 1 The MUN Explorer AUV

The following section describes some of the manoeuvring trials, particularly the acceleration - deceleration tests and turning circles in detail.

\section{MANOEUVRING TRIALS}

The manoeuvring trials were performed at Holyrood harbour which is situated $45 \mathrm{~km}$ south-west of St. John's, Newfoundland (Lat.=47.38849500 and Long. =-53.13486333). This sheltered body of water had sufficient depth and spread so as to carry out all the intended tests. The water depth ranged from 10 to $50 \mathrm{~m}$ and more. The vehicle was launched from a wharf thereby avoiding the need for a support vessel.

All missions were created using the software "FleetManager" - developed by the company Advanced
Concept and System Architecture (ACSA), France [5] and supplied with the vehicle by ISE. The mission plan files thus generated were later transferred to the VCC by means of a hardwire Ethernet link before the launch. This link was also used to retrieve the data from the VCC after all missions were completed. In order to avoid the effect of surface disturbances, all missions in a the horizontal plane were performed at a depth of $3 \mathrm{~m}$ below the water surface.

Manoeuvring, in naval architectural terms, can be defined as the controlled change in the direction of motion of a marine vehicle. Manoeuvring characteristics can be obtained by changing or maintaining a pre-defined course and speed of the vehicle in a systematic manner by means of working controls. In the case of the MUN Explorer AUV, these controls are tail planes, dive planes and the propeller.

The tail planes are arranged in an X-tail configuration as seen in Figures 1 and 2. In the discussions that follow, the control plane deflections play a crucial role in understanding the behaviour of the vehicle during a manoeuvre. Hence it is useful to have a brief explanation of the sign conventions used by the vehicle manufacturer and the consequent changes they bring about in the vehicle motion. This is explained through the following Figure and sketches.

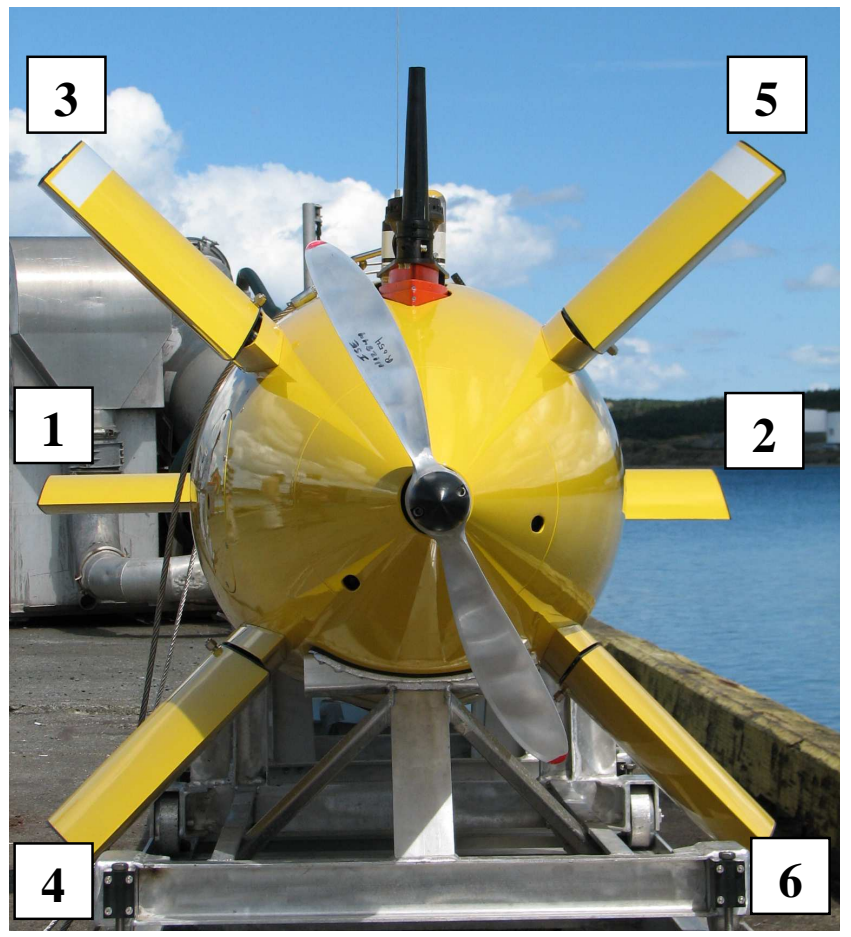

Fig. 2 The X-Tail Configuration with Plane Numbers

The sign convention adopted by the vehicle is shown in the sketch below in Figure 3a. The arrows show the positive direction of deflection of the trailing edge of the control plane. In other words, a positive deflection will be obtained by a right-hand-rule with the thumb pointing away from the body. Further, Figure $3 \mathrm{~b}$ shows the tail fin configuration for positive roll, pitch and yaw control of the vehicle. A vehicle with an X- 
tail configuration would use all four fins to manoeuvre in the horizontal or vertical plane unlike the cruciform ' + ' shape which for pitch and yaw control uses the horizontal and vertical fins respectively.

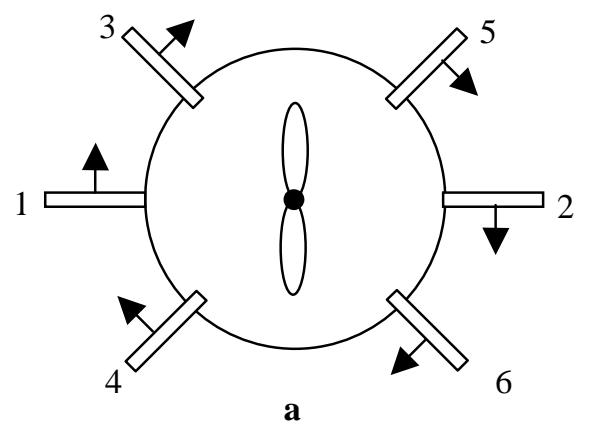

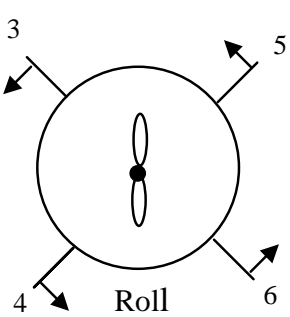

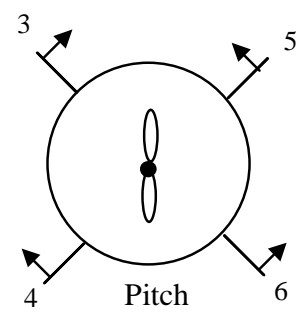

b

Fig. 3 Sketches Showing the Positive Sign Conventions

Keeping the sign convention, shown in Figure 3a, in mind, the positive pitch, yaw and roll control (shown in Figure 3b) can be defined in terms of individual control plane deflections as given by the following formulas [6]:

$$
\begin{aligned}
& \delta P=\frac{\delta_{3}+\delta_{4}-\delta_{5}-\delta_{6}}{4} \\
& \delta Y=\frac{\delta_{3}-\delta_{4}+\delta_{5}-\delta_{6}}{4} \text { and } \\
& \delta R=\frac{-\delta_{3}-\delta_{4}-\delta_{5}-\delta_{6}}{4}
\end{aligned}
$$

where $\delta \mathrm{P}, \delta \mathrm{Y}$ and $\delta \mathrm{R}$ stand for pitch, yaw and roll respectively. For instance, a positive value of $\delta \mathrm{Y}$ tends to turn a vehicle to starboard. The dive planes engage only in assisting pitch and roll motions of the vehicle and their combined effect can be expressed as:

$$
\delta P_{D}=\frac{-\delta_{1}+\delta_{2}}{2} \text { and } \delta R_{D}=\frac{-\delta_{1}-\delta_{2}}{2}
$$

This representation is useful for presenting the results and discussions that follow, particularly for the results from the turning circles.

\section{A. Acceleration / Deceleration Tests}

An acceleration test is performed by increasing the speed of vehicle from rest or from a particular ahead speed to a higher ahead speed while a deceleration test is performed by decreasing the speed of a vehicle from a particular speed to a lower value or allowing the vehicle to coast to rest [6]. Theoretically, an AUV is a neutrally buoyant vehicle. However, for practical reasons, an AUV is often designed to be slightly positively buoyant such that it becomes difficult or impossible to maintain the vehicle at rest at any depth except on the surface because the vehicle slowly rises to the surface. The MUN Explorer AUV is approximately $6 \mathrm{~kg}$ positively buoyant. This overruled the possibility of starting the vehicle from rest at a certain depth. Further, to make the vehicle dive to a depth of $3 \mathrm{~m}$, where all manoeuvres were performed, it was necessary to have the vehicle moving at a considerable speed so as to make the control planes effective, as these are the only means by which the AUV could dive or climb during a mission.

To overcome the above difficulties, it was decided to perform the test by changing the speed at regular intervals while the vehicle followed a straight line path at $3 \mathrm{~m}$ depth. Figure 4 shows the path of the vehicle during a straight-line

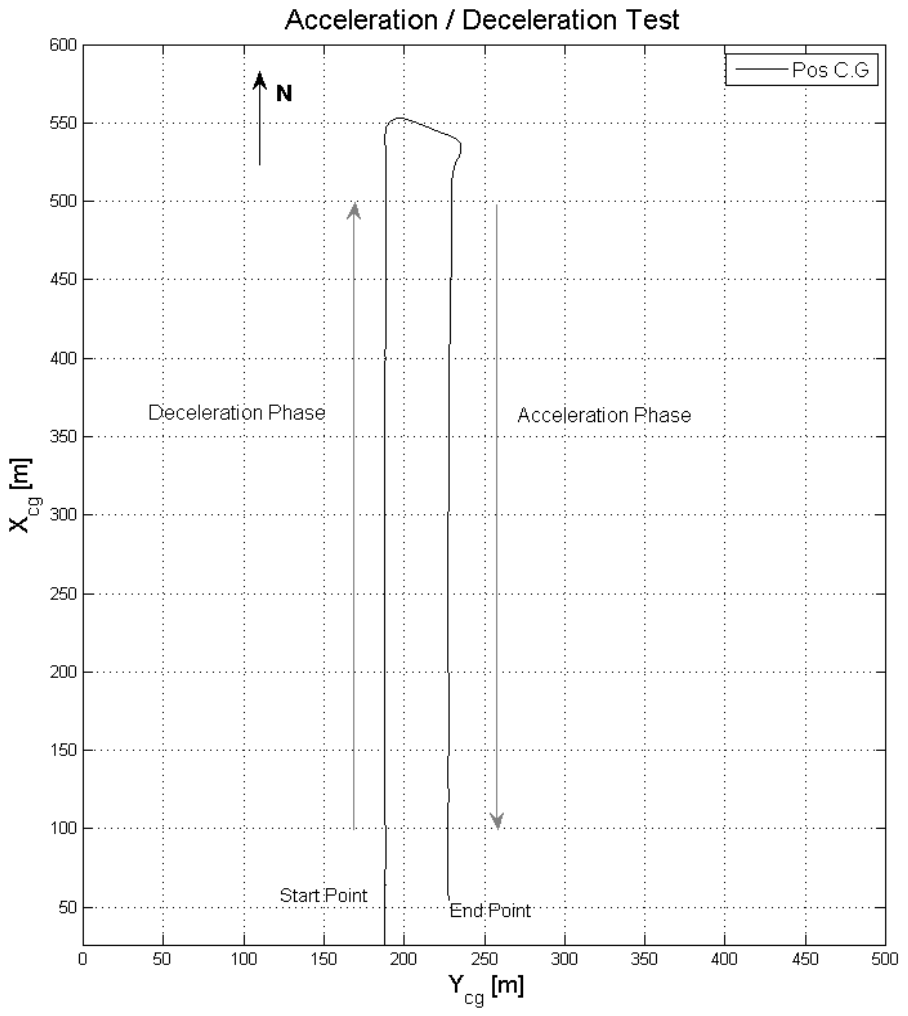

Fig. 4 Trajectory of the AUV from a straight-line test

test. Two tests were done in total. The vehicle was programmed to start from the surface at a speed of $1.5 \mathrm{~m} / \mathrm{s}$, which enabled the vehicle to dive to $3 \mathrm{~m}$ depth. Allowing enough time to reach the prescribed depth and to level off, the 
actual mission started by changing the speed at regular intervals. In Figure 4, the vehicle headed north in a straight line starting with a maximum set-speed of $2.5 \mathrm{~m} / \mathrm{s}$ and reduces it in steps of $0.5 \mathrm{~m} / \mathrm{s}$ finally reaching $1 \mathrm{~m} / \mathrm{s}$ towards the end of the line. This constitutes the deceleration phase. The vehicle took a turn to starboard at the end of the line and started the acceleration phase by heading south in a straight line. Here it started with a minimum set- speed of $1 \mathrm{~m} / \mathrm{s}$ and increased it in steps of $0.5 \mathrm{~m} / \mathrm{s}$ reaching a maximum of $2.5 \mathrm{~m} / \mathrm{s}$ towards the end of the line. Each speed was maintained for a length-oftravel of $100 \mathrm{~m}$ and at no time during the mission did the vehicle come to a complete stop.

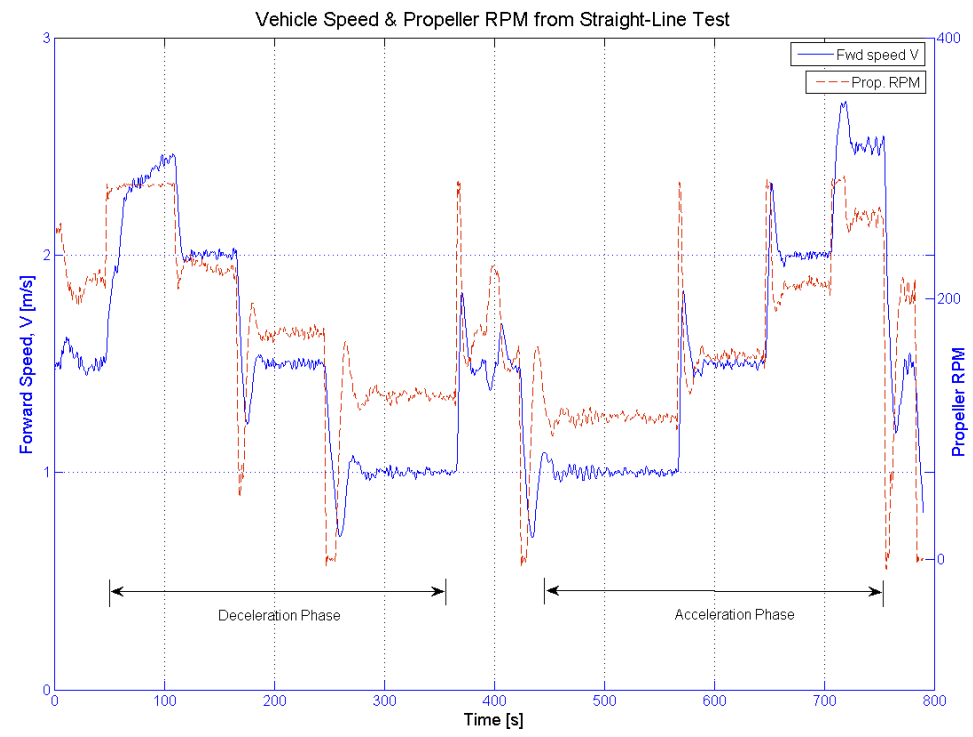

Fig. 5 Speed and Propeller RPM plot from straight-line test

The plot of forward speed, $\mathrm{V}$ and the corresponding propeller rpm for the above mission, which includes both the acceleration and deceleration phases, is shown in Figure 5. The first half corresponds to the deceleration phase of the run and the latter half corresponds to the acceleration phase of the run. A closer look at the Figure reveals that the propeller rpm for the acceleration phase is slightly lower than the propeller rpm for the deceleration phase and this difference can be seen predominantly at lower values of forward speed. This discrepancy may be due to the presence of a cross current whose in-line component has worked to the advantage of the vehicle in the deceleration phase.

The second test of this nature was performed with the same mission plan file thus making it a repetition of the first. The values of the velocity and rpm from both these tests are summarized in a single plot shown in Figure 6. It can be seen that the propeller rpm corresponding to higher speeds ( 2 and $2.5 \mathrm{~m} / \mathrm{s}$ ) almost coincide for both acceleration and deceleration phases while at lower speed they tend to be different. At lower speeds the vehicle may be more susceptible to an in-line currents.

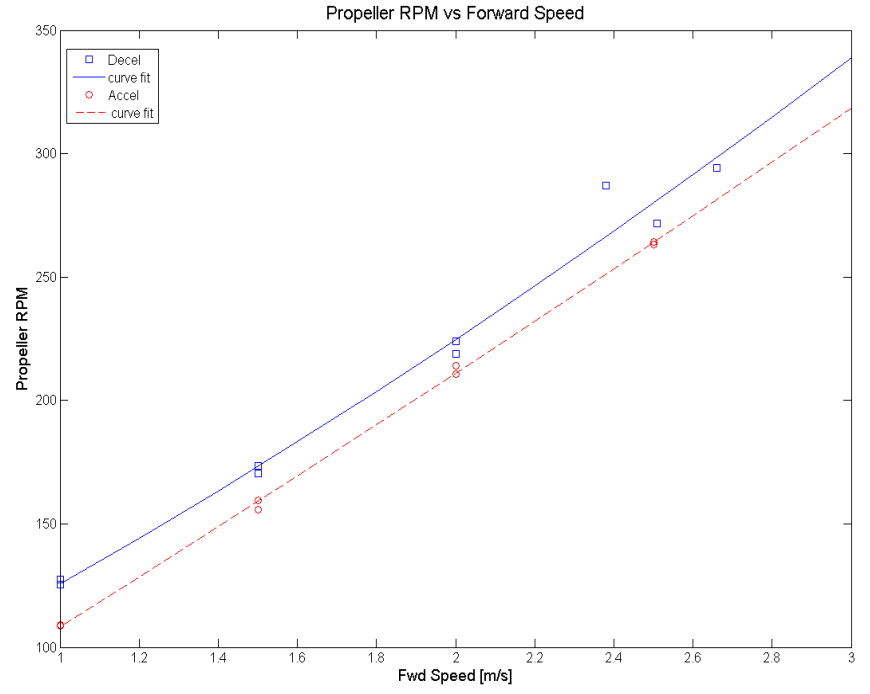

Fig. 6 Propeller RPM vs Speed for both acceleration and deceleration case

The control plane deflections corresponding to each forward speed for one complete run are shown in Table 1. In an ideal straight-line test, the control plane deflections should be zero or close to zero. However, from the Table, it can be seen that at lower speeds the deflections are significant. The effective individual control plane deflections $\delta \mathrm{P}, \delta \mathrm{Y}, \delta \mathrm{R}, \delta \mathrm{P}_{\mathrm{D}}$ and $\delta \mathrm{R}_{\mathrm{D}}$ were calculated using the expressions (1), (2) (3) and (4) respectively and are shown in Table 2 . For instance, $\delta \mathrm{P}$ for the case of $1 \mathrm{~m} / \mathrm{s}$ in the deceleration phase can be calculated as follows:

$$
\begin{aligned}
& \delta P=\frac{\delta_{3}+\delta_{4}-\delta_{5}-\delta_{6}}{4} \\
& =\frac{4.83+6.54-(-5.99)-(-4.89)}{4}=5.56^{\circ}
\end{aligned}
$$

TABLE I

CONTROL PLANE DEFLECTION ANGLES FOR STRAIGHT-LINE TESTS

\begin{tabular}{|c|c|c|c|c|c|c|c|}
\hline $\begin{array}{c}\text { V } \\
\mathrm{m} / \mathrm{s}\end{array}$ & $\begin{array}{c}\text { Dive Plane } \\
{[\mathrm{deg}]}\end{array}$ & \multicolumn{5}{c|}{$\begin{array}{c}\text { Tail Plane } \\
{[\mathrm{deg}]}\end{array}$} & $\begin{array}{c}\text { Prop } \\
\text { RPM }\end{array}$ \\
\hline & P1 & P2 & P3 & P4 & P5 & P6 & n \\
\hline \multicolumn{7}{|c|}{ Deceleration Phase } \\
\hline 2.4 & 1.71 & -2.13 & -0.80 & 0.47 & -2.89 & 0.01 & 287.0 \\
\hline 2.0 & 2.25 & -2.58 & 1.44 & 2.16 & -2.97 & -1.68 & 223.9 \\
\hline 1.5 & 0.60 & -0.64 & 2.12 & 3.59 & -3.81 & -3.11 & 173.4 \\
\hline 1.0 & -4.59 & 4.52 & 4.83 & 6.54 & -5.99 & -4.89 & 125.5 \\
\hline \multicolumn{7}{|c|}{ Acceleration Phase } \\
\hline 1.0 & -7.09 & 6.96 & 7.63 & 8.77 & -6.91 & -5.83 & 109.0 \\
\hline 1.5 & -1.31 & 1.18 & 2.73 & 3.99 & -4.17 & -3.17 & 155.8 \\
\hline 2.0 & 1.47 & -1.67 & 0.79 & 1.68 & -2.65 & -1.78 & 210.8 \\
\hline 2.5 & 0.46 & -0.87 & 0.10 & 1.08 & -1.37 & -0.75 & 263.2 \\
\hline
\end{tabular}


A positive value for $\delta \mathrm{P}$ or $\delta \mathrm{Y}$ or $\delta \mathrm{R}$ implies that the given combination of control plane deflections will tend to produce a positive change in attitude. For instance, a positive value of $\delta \mathrm{Y}$ implies that the combination of control plane deflections would cause the vehicle to turn starboard. Further, as mentioned before, the dive planes are also effective in providing pitch and roll control. Their combined effect is given by the values $\delta \mathrm{P}_{\mathrm{D}}$ and $\delta \mathrm{R}_{\mathrm{D}}$. Table 2 also shows the measured pitch and roll attitudes of the vehicle at each forward speed.

TABLE 2

EFFECTIVE CONTROL PLANE ANGLES

\begin{tabular}{|c|c|c|c|c|c|c|c|}
\hline $\mathbf{V}$ & $\boldsymbol{\delta}_{\mathbf{D}}$ & $\boldsymbol{\delta}_{\mathbf{D}}$ & $\boldsymbol{\delta P}$ & $\boldsymbol{\delta} \mathbf{Y}$ & $\boldsymbol{\delta R}$ & Roll & Pitch \\
\hline $\mathrm{m} / \mathrm{s}$ & deg & deg & deg & $\operatorname{deg}$ & $\operatorname{deg}$ & $\phi$ & $\theta$ \\
\hline \multicolumn{7}{|c|}{ Deceleration Phase } \\
\hline 2.38 & -1.92 & 0.21 & 0.64 & -1.04 & 0.804 & 0.019 & 0.017 \\
\hline 2.00 & -2.41 & 0.17 & 2.07 & -0.50 & 0.264 & 0.006 & -0.042 \\
\hline 1.50 & -0.62 & 0.02 & 3.16 & -0.54 & 0.301 & 0.009 & -1.183 \\
\hline 1.00 & 4.55 & 0.03 & 5.57 & -0.70 & -0.123 & -0.004 & -4.055 \\
\hline \multicolumn{7}{|c|}{ Acceleration Phase } \\
\hline 1.00 & 7.03 & 0.06 & 7.28 & -0.55 & -0.914 & 0.005 & -6.167 \\
\hline 1.50 & 1.24 & 0.07 & 3.51 & -0.56 & 0.155 & 0.014 & -1.750 \\
\hline 2.00 & -1.57 & 0.10 & 1.73 & -0.44 & 0.489 & 0.020 & -0.068 \\
\hline 2.50 & -0.67 & 0.21 & 0.83 & -0.40 & 0.233 & -0.051 & -0.030 \\
\hline
\end{tabular}

It is observed from Table1 that slower speeds required larger dive plane deflections in order to maintain level flight at constant forward speed. The AUV seems to be very well roll-stabilized. However, in Table-2, the angles $\delta \mathrm{P}_{\mathrm{D}}$ and $\delta \mathrm{P}$ are positive and significantly higher for lower speed runs than for the higher speeds. Despite all six planes working hard to bring the vehicle to a nose-up attitude, the pitch attitude of the vehicle is seen to be negative. This could be explained by the fact that when the vehicle is at rest, it is normally trimmed to have the nose down and with a small starboard heel. Nose down (i.e. tail up) trim is to ensure that the antennas mounted on the communications mast will be well out of the water when the AUV is at rest at the surface thus providing a failsafe condition for communications. Starboard heel trim is to compensate for the effect of propeller torque such that at design speed $(1.5 \mathrm{~m} / \mathrm{s})$, the vehicle will be running upright at zero heel. As the vehicle speed with respect to water decreases, the amount of force generated by each control plane is decreased until a point is reached where the net forces are not enough to overcome the vehicle's positive buoyancy and trim. This possibly explains the reason for an observed negative pitch attitude despite positive plane deflections during slow forward speed runs, whereas at higher speeds the planes generate enough force to overcome the innate nose-down pitch attitude. Further, the drift from the intended straight line was noted to be very little with the AUV drifting only $0.13 \%$ of the distance travelled in its deceleration phase and $0.41 \%$ of the distance travelled in its acceleration phase with much of it occurring during the lower speed regime.

TABLE 3

MEASURED AND ESTIMATED PARAMETERS FOR TURNING CIRCLES AT A SPEED OF $1 \mathrm{~m} / \mathrm{s}$

\begin{tabular}{|c|c|c|c|c|c|c|c|c|c|c|c|c|c|c|c|c|c|c|}
\hline \multirow{3}{*}{ No. } & \multicolumn{6}{|c|}{ Individual Control Plane Angles [deg] } & \multicolumn{5}{|c|}{ Effective Plane Angles [deg] } & \multirow{3}{*}{$\begin{array}{c}\text { Prop. } \\
\text { RPM } \\
\mathbf{n} \\
\end{array}$} & \multirow{3}{*}{$\begin{array}{c}\text { Speed } \\
\mathrm{m} / \mathrm{s} \\
\mathbf{U}_{\mathbf{t}}\end{array}$} & \multicolumn{2}{|c|}{ Ang. Disp [deg] } & \multirow{3}{*}{$\begin{array}{c}\text { Radius } \\
{[\mathrm{m}]} \\
\mathbf{R} \\
\end{array}$} & \multicolumn{2}{|c|}{ Turning rates } \\
\hline & \multicolumn{2}{|c|}{ Dive Planes } & \multicolumn{4}{|c|}{ Tail Planes } & \multicolumn{2}{|c|}{ Dive Planes } & \multicolumn{3}{|c|}{ Tail Planes } & & & Roll & TITI & & $\mathrm{rad} / \mathrm{s}$ & $\mathrm{r} . \mathrm{L} / \mathrm{U}_{\mathrm{t}}$ \\
\hline & P1 & $\mathbf{P 2}$ & $\mathbf{P 3}$ & $\mathbf{P 4}$ & P5 & P6 & $\delta \mathbf{P}_{\mathrm{D}}$ & $\delta \mathbf{R}_{\mathbf{D}}$ & $\delta P$ & $\delta \mathbf{Y}$ & $\delta \mathbf{R}$ & & & $\phi$ & $\boldsymbol{\theta}$ & & $r$ & $r^{\prime}$ \\
\hline 1 & -4.17 & 17 & .32 & 10 & . & 12.51 & 17 & 0 & 6.19 & 5.23 & 8 & 121.4 & 1.01 & 0.001 & -4.809 & 22.51 & 2.560 & 0.1999 \\
\hline 2 & -4.71 & 1 & .54 & -0.01 & 6 & -12.52 & 71 & & 3 & & 6 & 9 & 00 & 5 & -4 & 0 & 13 & 91 \\
\hline 3 & 23 & 29 & 10.19 & 0.34 & -2.38 & -12.18 & 26 & -0.03 & 7 & 91 & 1 & 4 & 1.01 & 0.001 & -5.061 & 25.02 & 4 & 9 \\
\hline 4 & -5.04 & 506 & & 0.39 & -2.08 & -12.26 & 5.05 & -0.01 & 6.26 & & 0.91 & 1.9 & 1.01 & 0.001 & -4. & 5.09 & 296 & 1794 \\
\hline 5 & -5.12 & 1 & 1017 & 0.09 & -2.43 & -11.82 & 5.12 & 0.00 & 3 & 7 & 0.80 & 121.6 & 1.01 & -0.003 & -5.079 & 26.58 & 2.180 & 0.1693 \\
\hline 6 & -5.36 & 36 & 010 & 0.97 & -2.70 & -11.54 & 36 & 0.00 & 6.33 & 4.49 & 0.79 & 6 & 1.00 & 1 & -5 & 27.97 & 3 & 99 \\
\hline 7 & 28 & 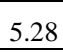 & 9.87 & 1.23 & -2.77 & -11.60 & 5.28 & 0.00 & 1 & 5 & 2 & 6 & 1.02 & 1 & 3 & 1 & 070 & 1601 \\
\hline 8 & 94 & & & & 2.00 & -1131 & & & 6.38 & & & 118.6 & 1.01 & \begin{tabular}{|l}
-0.003 \\
\end{tabular} & \begin{tabular}{|l|}
-5.095 \\
\end{tabular} & 29.65 & 1.954 & 0.1518 \\
\hline 9 & \begin{tabular}{|l}
-5.42 \\
\end{tabular} & 2 & & 2 & \begin{tabular}{|lll}
-3 & \\
\end{tabular} & -10.51 & 47 & -0.04 & 6.62 & 3.46 & 0.40 & 2 & 99 & 2 & -5. & 33.44 & 695 & 346 \\
\hline 10 & .82 & 5.83 & 9.41 & 2.77 & -3.70 & -10.34 & 83 & 0.00 & 6.56 & 3.32 & 0.47 & 121.8 & 1.00 & 0.003 & -5.358 & 37.54 & 1.531 & 0.1199 \\
\hline
\end{tabular}




\section{B. Turning Circles}

The next set of tests completed were the turning circles. The turning circle is a steady-state manoeuvre in which the vehicle enters a steady turn at a constant speed. It is traditionally performed by deflecting the rudders to a pre-defined angle and holding them fixed resulting in the vehicle entering a circular path. The manoeuvre is also an indicator of the efficiency of the rudder. The control inputs for a turning circle manoeuvre are usually the rudder angle and the forward speed but in these tests the control inputs were forward speed and the desired radius of turn. Here again, the missions were performed at a depth of $3 \mathrm{~m}$ and at three different forward speeds: $1,1.5 \& 2$ $\mathrm{m} / \mathrm{s}$. For brevity, in the discussions that follow, only the case of $1 \mathrm{~m} / \mathrm{s}$ speed is shown. The following Figure shows the trajectory of the vehicle during a particular turn; the commanded speed was $1 \mathrm{~m} / \mathrm{s}$.

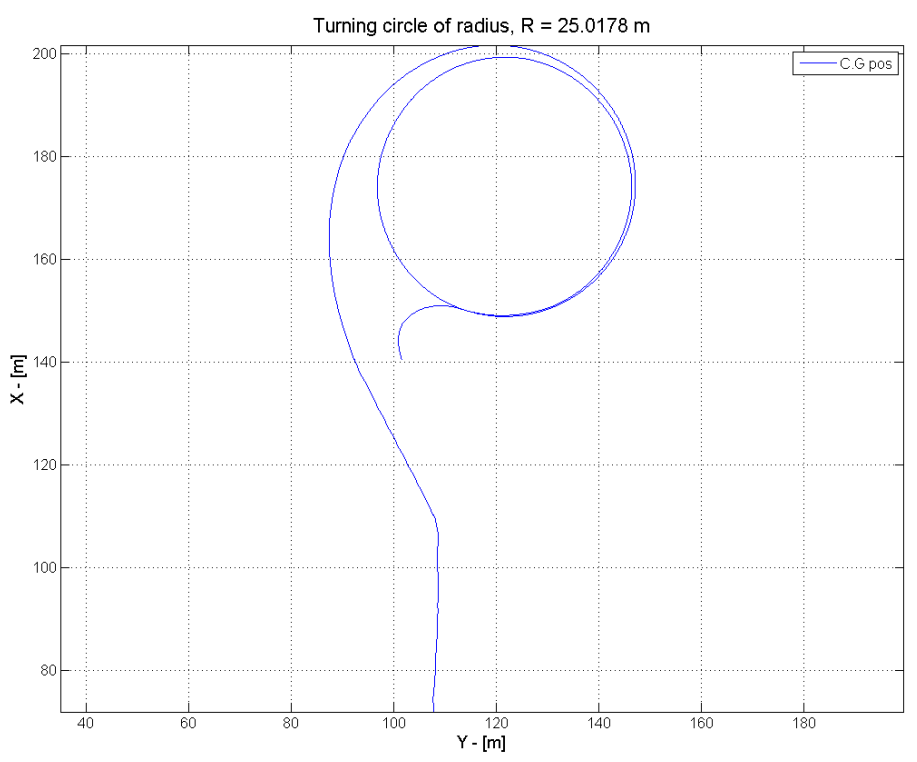

Fig. 7 Turning circle at a speed of $1 \mathrm{~m} / \mathrm{s}$

In order to understand how effective the control planes are in turning the vehicle, a set of circles were performed at the same speed (e.g. $1 \mathrm{~m} / \mathrm{s})$. As all four tail-planes are involved in turning the vehicle it is difficult to plot the relationship between the tail-plane angles and corresponding radius in a single plot. A better approximation would be to represent the effective yaw-angle-of-turn produced by the four planes, by means of the expression for $\delta Y$. The same can be calculated for pitch and roll controls as done earlier in the previous subsection. These values are shown in Table 3. The Table also shows the pitch and roll displacements along with propeller rpm and steady-state forward speed. All turns were performed to the starboard side (as in Fig. 7). Note that the values of $\delta Y$ are all positive indicating starboard turns. These values of $\delta Y$ were used to plot the variation of turning radius with plane angles as shown in Figure 8.

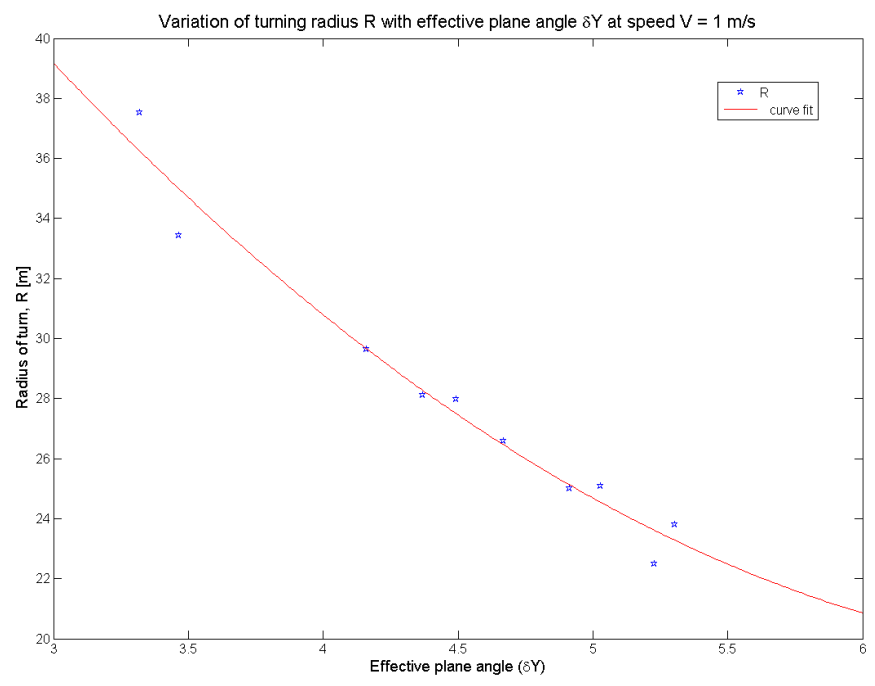

Fig. 8 Effect of Control Plane Deflection on Turning Radius

The plot shows a decreasing radius of turn with increasing tail-plane deflections, which would be normally expected. In the case of a starboard turn, when the control planes are deflected initially, the force acting on the planes tends to push the aft end of the vehicle to port from its original straight-line course. As the moment due to this force turns the vehicle's head, the lateral force on the hull builds up helping the vehicle to turn to starboard. Once the vehicle enters a steady turn, some other parameters also become of interest. They are the drift angle, the turning rate, the speed loss during the turn (or steadystate speed) and the heel angle during the turn.

When a vehicle enters a steady turn, the centrifugal force acting through the center of gravity (C.G) of the vehicle counteracts the radial component of the hydrodynamic force acting towards the center of the circle. The location of the point of application of these forces produces an inward heel angle for a submerged vehicle. This would be prominent in vehicles with a sail or similar structures such as in submarines. Since the MUN Explorer AUV is axisymmetric and without any sail, the vehicle is not expected to exhibit much roll during a steady turn. From Table 3 , it is seen that the roll angle $\phi$ during a steady turn is negligibly small. The near zero values of $\delta R_{D}$ imply that the dive planes are hardly working to bring about any roll stabilization while the tail planes may be assisting with the roll stabilization. This is confirmed when we look at the individual tail plane angles given in Table 3 , where planes 4 and 5 have deflections of opposite configurations (signs) compared to the sign conventions shown in Figure $3 \mathrm{~b}$ for 'Yaw'. Regardless, the values for $\delta \mathrm{R}$ are seen to be slightly positive which must be present to counteract the torque from the propeller. The pitch angles $\theta$, however, for this set of circles at $1 \mathrm{~m} / \mathrm{s}$ are considerably higher (approx. $-5^{\circ}$ ) just as in the case of the acceleration-deceleration test. As explained before, this happens as a result of the slow manoeuvring speed where the 
control planes are less effective in generating enough force to counter the inherent nose-down pitch.

In order to generate a radial force of sufficient magnitude to turn the vehicle, the hull should be held at an angle relative to the flow. The rudder forces should be capable of holding the vehicle at this angle of attack, called the drift angle, $\beta$. It is defined as the angle between the centerline of the vehicle and the tangent to the path at the point concerned, the C.G in this case. Figure 9 shows the variation of drift angle $\beta$ with effective plane angle, $\delta Y$ for turning circles performed at a speed of $1 \mathrm{~m} / \mathrm{s}$. The trend seems consistent with $\beta$ increasing as $\delta Y$ increases which means that the drift angle increases as the radius of turn decreases. Another factor is the rate at which the heading changes during a turn, that is, the turning rate $r$. It is related to the speed of the vehicle and the radius of turn as $r=$ $U / R$, where $U$ is the tangential velocity and $R$ is radius of turn. The non-dimensional turning rate is given by:

$$
r^{\prime}=r \cdot \frac{L}{U}=L / R
$$

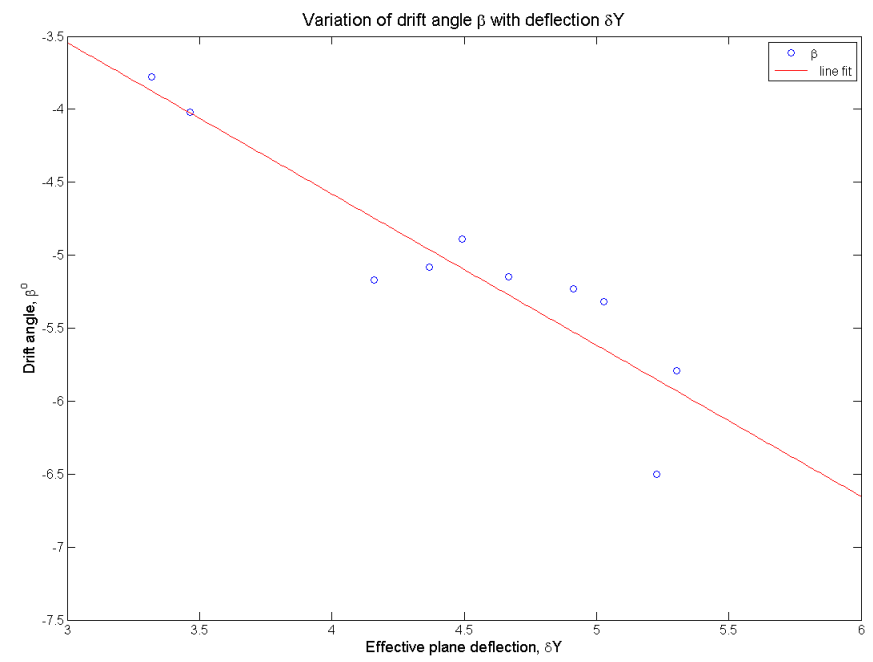

Fig. 9 Drift Angle $\beta$ variation with $\delta Y$ for circles at $\mathrm{V}=1 \mathrm{~m} / \mathrm{s}$

The non-dimensional turning rate $r$ ', from expression (5), thus becomes a function of just radius $R$ alone, as the overalllength of the vehicle $L$ remains a constant. Hence, $r$ ' increases as the radius of turn $R$ decreases. In order for the radius of turn to decrease, the yaw angle $\delta \mathrm{Y}$ has to increase (see Fig. 8). This explains the trend seen in Figure 10 that an increase in $\delta Y$ increases $r$ '.

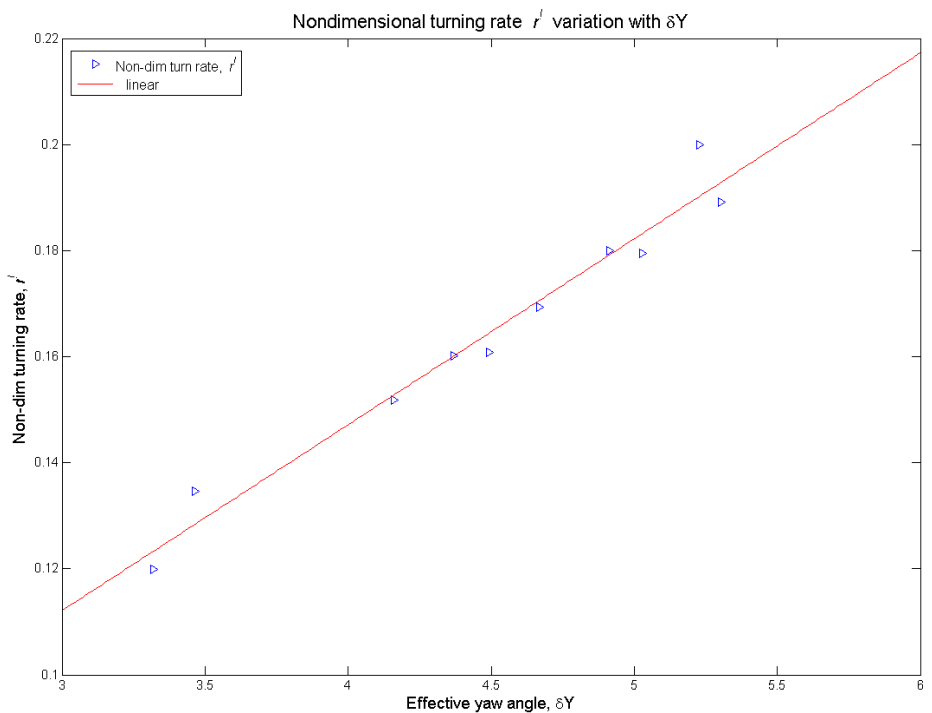

Fig. 10 Non-dimensional turning rate $r$ ' versus plane deflection $\delta \mathrm{Y}$

As mentioned above, the rudder holds the hull at an angle of attack in order to develop the force and moment necessary to turn the vehicle. This comes at a cost of increased drag. As a result of increased drag, a vehicle moving at constant speed would suffer a loss of speed when it enters a steady turn. The vehicle decelerates to a point until it reaches a new steady speed, which is less than the actual speed during a straight course with the propeller running at constant rpm. Surprisingly, from Table 3 , the tangential velocity $U_{t}$, during the steady-state turns appears equivalent to the commanded forward speed of $1 \mathrm{~m} / \mathrm{s}$. A plot of the time series of speed and rpm for the particular circle in Fig. 7 is shown in Fig. 11. It is observed that the propeller rpm remains fairly constant almost through the entire run. Theoretically, it is not possible to have the same speed on a straight course and during a turn without actually changing the propeller rpm. But this pattern was consistent and was observed for all the tests shown in Table 3.

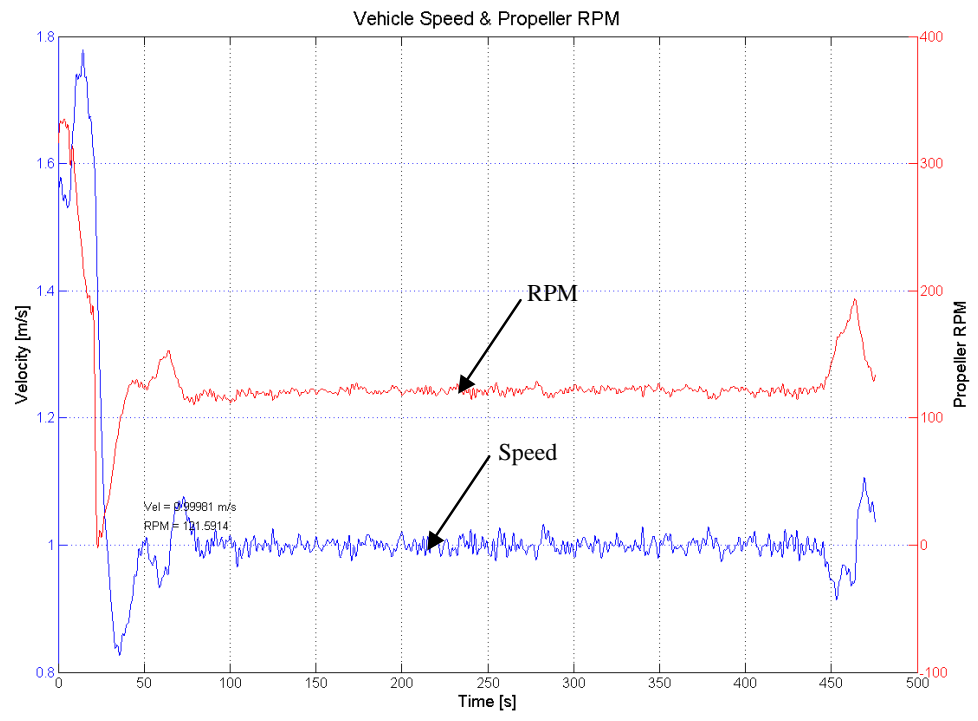

Fig. 11 Plot showing the time series of propeller rpm and corresponding speed for circle in Fig. 7 
A comparison between the propeller rpm from Table 3 and from Table 1for the straight-line test, for a speed of $1 \mathrm{~m} / \mathrm{s}$, does not give any distinct information leaving the issue inconclusive. It may be that since the radius of turn is large with a diameter-to-length $(\mathrm{D} / \mathrm{L})$ ratio of 10 and more, the estimated drift angle experienced by the AUV may generate drag not much different from that during a straight course. This also shows the ability of the control system to maintain a command speed all through the turn thus ensuring consistent sampling conditions for any flow through instruments such as the CTD (conductivity, temperature and depth) sensor.

\section{CONCLUSIONS}

The results obtained from acceleration - deceleration tests and turning circles provides information about the general performance of the vehicle. The main purpose of these experiments was to collect some useful data for validation of a hydrodynamic model. However, the data set also formed a basis for understanding the inherent behaviour of the vehicle that might be useful in future design changes. From the acceleration-deceleration test, it was observed that slower speeds required larger dive plane deflections in order to maintain level flight at constant forward speed. This was the same case for turning circles at $1 \mathrm{~m} / \mathrm{s}$ speeds. The straight-line test also demonstrated the ability of the vehicle in following a predefined path with minimum drift. The set of turning circles performed at a speed of $1 \mathrm{~m} / \mathrm{s}$ were presented. From both straight-line and turning circle tests it was evident that the vehicle had good roll stability; even when it is executing a turn. This is an essential quality of a sensor platform. It was also observed that the AUV maintained the commanded speed throughout the turn without any appreciable change in the propeller rpm. The exact reason for this behaviour is uncertain though. During future experiments, it would be more appropriate to use constant propeller rpm as opposed to constant speed as the control input to the vehicle, so that any change in speed during a turn or dive would be clearly evident. Further, some of the information gained from these experiments such as the relationship between speed and the corresponding propeller rpm can be used in the hydrodynamic formulation as a look-up table to enhance the modeling of propeller thrust. The rudder angle and forward speed information obtained for turning circles are useful as inputs to the hydrodynamic simulation model.

\section{ACKNOWLEDGMENTS}

The first three authors thank the Natural Sciences and Engineering Research Council (NSERC) for the funding to support this research. Thanks are also given to the Canadian Fund for Innovation and Atlantic Canada Opportunities Agency (ACOA) through an Atlantic Innovation Fund award to the Pan Atlantic Petroleum Systems Consortium for funding the instrumentation and student funding. The team also thanks the International Submarine Engineering (ISE) for their continuing technical support.

\section{REFERENCES}

[1] M. Nahon, 1996, "A Simplified Dynamics Model for Autonomous Underwater Vehicles", Autonomous Underwater Vehicle Technology, AUV '96, pp. 373 - 379

[2] D. E. Perrault, 2002, "Autonomous Underwater Vehicles (AUVs) Sensitivity of Motion Response to Geometric and Hydrodynamic Parameters and AUV Behaviors with Control Plane Faults", Ph.D. Thesis, Memorial University of Newfoundland, February 2002.

[3] J. Evans and M. Nahon, 2004, "Dynamic Modeling and Performance Evaluation of Autonomous Underwater Vehicle, Ocean Engineering, 31, pp. $1835-1858$.

[4] M.T. Issac, S. Adams, M. He, N. Bose, C. D. Williams, R. Bachmayer, T. Crees, "Manoeuvring Experiments Using the MUN Explorer AUV", International Symposium on Underwater Technology, Tokyo, Japan, 17 - 20 April, 2007.

[5] A.C.S.AUnderwater GPS, http://www.underwater-gps.com

[6] M. J. Hemsch and J. N. Nielsen, Tactical Missile Aerodynamics, Progress in Astronautics and Aeronautics, Volume 104.

[7] E. V. Lewis, Principles of Naval Architecture, Vol. III, p $251-256$. 